\title{
Long-Term Safety and Efficacy of Tacrolimus in Myasthenia Gravis
}

\author{
Yool-hee Kim ${ }^{1}$, Ha Young Shin ${ }^{2}$, and Seung Min Kim² \\ ${ }^{1}$ Department of Neurology, College of Medicine, Korea University Ansan Hospital, Ansan; \\ ${ }^{2}$ Department of Neurology, Yonsei University College of Medicine, Seoul, Korea.
}

Purpose: Myasthenia gravis (MG) is a lifelong autoimmune disorder that affects neuromuscular transmission. The long-term treatment plan should include immunotherapy. We investigated the long-term safety and efficacy of tacrolimus for the treatment of MG in real-world clinical practice.

Materials and Methods: We retrospectively reviewed $160 \mathrm{MG}$ patients treated with tacrolimus from 2005 to 2015. Myasthenia Gravis Foundation of America (MGFA) clinical classification, MGFA post-intervention status, myasthenic functional score, and dose of oral prednisolone were investigated.

Results: Adverse events occurred in 68 patients (42.5\%), most of which were minor and well-managed. Clinical severity scales improved after administration of tacrolimus, compared to the baseline. Compared to 6 months before administration of tacrolimus, prednisolone dose significantly decreased at 12 months after treatment (2.85 $\pm 0.92 \mathrm{mg} /$ day, $p=0.002), 18 \mathrm{months}$ after treatment (3.36 $\pm 0.99 \mathrm{mg} /$ day, $p=0.001)$, and 24 months after treatment $(3.71 \pm 0.93 \mathrm{mg} / \mathrm{day}, p<0.001)$.

Conclusion: Tacrolimus may be effective in reducing the severity of MG and may permit a reduction in the steroid dose prescribed to the patients. Adverse events due to tacrolimus treatment were not serious.

Key Words: Myasthenia gravis, tacrolimus, steroid

\section{INTRODUCTION}

Myasthenia gravis (MG) is an autoimmune disorder that affects neuromuscular transmission as a result of antibodies binding to elements of the neuromuscular junction, most commonly the acetylcholine receptor (AChR). ${ }^{1,2}$ The prevalence of MG is increasing in South Korea ${ }^{3,4}$ and many patients suffer from uncontrolled symptoms. In recent years, the prognosis for patients with MG has generally been good. One major reason for this improved prognosis is the increasing availability of immunomodulating drugs. ${ }^{5}$

\footnotetext{
Received: September 4, 2018 Revised: March 8, 2019

Accepted: April 9, 2019

Corresponding author: Ha Young Shin, MD, Department of Neurology, Yonsei University College of Medicine, 50-1 Yonsei-ro, Seodaemun-gu, Seoul 03722, Korea. Tel: 82-2-2228-1600, Fax: 82-2-393-0705, E-mail: hayshin@yuhs.ac

-The authors have no potential conflicts of interest to disclose.

(C) Copyright: Yonsei University College of Medicine 2019

This is an Open Access article distributed under the terms of the Creative Commons Attribution Non-Commercial License (https://creativecommons.org/licenses/ by-nc/4.0) which permits unrestricted non-commercial use, distribution, and reproduction in any medium, provided the original work is properly cited.
}

Tacrolimus is a calcineurin inhibitor and inhibits T cell activation. ${ }^{6-8}$ It was originally used to prevent rejection after organ transplantation. Since successful treatment of MG with tacrolimus was reported in 2002, ${ }^{9}$ it has been used to treat MG. Because MG is a lifelong medical condition, tacrolimus, like other immunomodulating drugs, should be used as part of a long-term plan. Although a number of studies have assessed tacrolimus use in $\mathrm{MG},{ }^{10-22}$ the long-term safety and efficacy of tacrolimus in MG is not entirely understood. ${ }^{21}$ In addition, most observational studies have investigated the efficacy of tacrolimus by comparing steroid doses and clinical severity at the start of tacrolimus administration with those after treatment. However, this comparison is not appropriate because MG has a fluctuating clinical course and the addition of immunosuppressants like tacrolimus may be made during clinical deterioration and may accompanied by an increase in steroid doses. Although two previous studies investigated clinical status and steroid doses 4 weeks before the start of tacrolimus, the small number of patients included (19 and 9 patients) was too few for generalization and the four-week period may be insufficient given the long clinical course of MG. ${ }^{10,17}$ 
The purpose of this study was to investigate the long-term safety and efficacy of tacrolimus in the treatment of MG by analyzing clinical status and steroid doses before and after tacrolimus treatment in real-world clinical practice.

\section{MATERIALS AND METHODS}

This was a retrospective study including all patients diagnosed with MG and treated with tacrolimus from December 2005 to July 2015 at Severance Hospital, Yonsei University Health System. The requirement to obtain informed consent from study subjects was waived by the Institutional Review Board (IRB) of Severance Hospital, Yonsei University College of Medicine, Seoul, Korea (IRB No.: 4-2015-0322).

A diagnosis of MG was based on the signs and symptoms of muscle fatigue with at least one of the following: 1) $10 \%$ or more decrease in compound muscle action potential in response to low-frequency supramaximal repetitive nerve stimulation with five stimuli in a train, 2) positive serum antibodies against AChR or muscle-specific tyrosine kinase (MuSK), or 3) improvement in muscle fatigue after an intramuscular injection of neostigmine. Generally, the initiating dose of tacrolimus was $3 \mathrm{mg}$ once daily. If the effects were insufficient, dosage was increased to reach a trough level between $7-10 \mathrm{ng} / \mathrm{dL}$. If the patient complained of adverse events, the dose was decreased. Immunosuppressive drugs other than prednisolone were not used in combination with tacrolimus.

In total, $160 \mathrm{MG}$ patients were identified. Medical records were reviewed to collect information on age, sex, age at symptom onset, tacrolimus administration and discontinuation, thymus histopathology, Myasthenia Gravis Foundation of America (MGFA) clinical classification, MGFA post-intervention status (PIS), ${ }^{23}$ myasthenic functional score (MFS), ${ }^{24}$ and MG treatment, including corticosteroids, immunosuppressants, pyridostigmine, and thymectomy.

MFS is an indicator of MG severity and was first used in a trial comparing azathioprine to prednisolone. ${ }^{24}$ It is a five-grade functional scale, defined as 1) complete remission; 2) minor symptoms allowing normal activity, except for exertional activity; 3) moderate symptoms allowing occupational or partial daily activity; 4) major disability requiring discontinuation of occupational activity or major reduction of daily activity; and 5) major disability requiring continuous help from others or mechanical ventilation. ${ }^{24}$

\section{Safety}

Symptoms, medical co-morbidities, and laboratory analyses, such as blood urea nitrogen (BUN), creatinine (Cr), aspartate transaminase, alanine transaminase, phosphate, magnesium, sodium, potassium, chloride, lipid profile, fasting glucose, erythrocyte sedimentation rate, and C-reactive protein, were routinely monitored, and the results were collected from medi- cal records. Experienced neurologists reviewed adverse effects with laboratory tests for all patients, including tests for complete blood count, serum chemistry, and electrolytes.

\section{Efficacy}

To evaluate the efficacy of tacrolimus, MGFA clinical classification, MGFA PIS, MFS, and dose of oral prednisolone were evaluated. Data were analyzed at the initiation of tacrolimus treatment and at 6, 12, 18, and 24 months before and after treatment initiation. In patients who were treated with tacrolimus for more than 24 months, data were also retrieved from the last visit when tacrolimus was prescribed.

\section{Statistics}

Oral prednisolone doses were compared at different time points. A linear, mixed, repeated measures covariance model with unstructured covariance was used. Additionally, post hoc analyses were performed to compare prednisolone doses at each time point. We used Bonferroni correction for the multiple outcomes. Statistical analyses were performed using IBM SPSS Statistics for Windows, version 23 (IBM Corp., Armonk, NY, USA).

\section{RESULTS}

\section{Demographic characteristics}

Patient demographic characteristics are summarized in Table 1. A total of $160 \mathrm{MG}$ patients (124 females, 36 males) were identified. Females were more likely to be started on tacrolimus than males, mainly owing to their intolerance of hirsutism and gingival hyperplasia caused by the previous immunosuppressant (cyclosporine). The mean duration of MG at tacrolimus initiation was $10.8 \pm 8.4$ years. Anti-AChR antibody was detected in 140 (87.5\%) patients, and anti-MuSK antibody was positive in seven $(4.4 \%)$ patients. Thymectomy was performed in 87 (54.4\%) of the 160 patients. Thymoma was associated in 40 patients. At tacrolimus treatment initiation, MG severity according to MGFA clinical classification was mild (MGFA I or II) in 95 patients and more severe (MGFA III or IV) in 58 patients. Seven patients were free of MG symptoms and started tacrolimus due to steroid side effects (4), cyclosporine side effects (2), and steroid tapering failure (1) at the initiation of tacrolimus treatment (Table 1).

\section{Treatment with tacrolimus}

The mean duration of treatment with tacrolimus was $16.48 \pm$ 17.93 months (range 0-71 months). The median tacrolimus dose was $3.3 \pm 0.8 \mathrm{mg}$. In 52 patients who had been treated with prednisolone with or without pyridostigmine bromide, tacrolimus was added to prednisolone. In 99 patients who had been treated with prednisolone and immunosuppressants with or without pyridostigmine bromide, tacrolimus replaced the pre- 
Yool-hee Kim, et al.

Table 1. Demographic Features of 160 MG Patients

\begin{tabular}{ll}
\hline \multicolumn{1}{c}{ Characteristic } & \multicolumn{1}{c}{ Result } \\
\hline Age at onset (yr) & $31.9 \pm 15.7$ \\
Sex (male/female) & $36(22.5) / 124$ (77.5) \\
Age at tacrolimus administration (yr) & $43.2 \pm 13.8$ \\
Duration of MG at tacrolimus administration (yr) & $10.8 \pm 8.4$ \\
Thymectomized patients & 87 (54.4): normal thymus 12, hyperplasia 30, thymoma 40, involution 2, unknown 3 \\
Anti-AChR antibody & $140(87.5)$ \\
Anti-MuSK antibody (seropositive/borderline) & $7(4.4) / 2$ (1.3) \\
MGFA at tacrolimus administration (n) & I (39), Ila (26), Illb (30), Illa (27), IIIb (16), IVa (8), IVb (7), V (0), symptom free (7) \\
Duration of tacrolimus therapy (month) & $16.48 \pm 17.93$ (range 0-71) \\
Dose of tacrolimus (mg) & $3.3 \pm 0.8$ (range 2-8) \\
& Azathioprine (3), azathioprine+prednisolone (34), cyclosporine (6), cyclosporine+prednisolone (58), \\
Long-term immunotherapy before tacrolimus treatment (n) & azathioprine+cyclosporine+prednisolone (4), mycophenolate mofetil+prednisolone (3), \\
& prednisolone (52)
\end{tabular}

MG, myasthenia gravis; AChR, acetylcholine receptor; MuSK, muscle-specific tyrosine kinase; MGFA, Myasthenia Gravis Foundation of America. Variable are presented as mean \pm SD or number (percentage) unless otherwise noticed.

vious immunosuppressants. In nine patients who had been treated with immunosuppressants with or without pyridostigmine bromide, tacrolimus replaced the previous immunosuppressant. Among 108 patients who were using immunosuppressants other than tacrolimus, 50 changed to tacrolimus due to adverse effects from the previous immunosuppressant. The previous immunosuppressant therapies other than prednisolone are listed in Table 1. In 105 (65.6\%) of the 160 patients, tacrolimus was started during an exacerbation of MG.

\section{Safety}

Adverse events that were possibly associated with tacrolimus are presented in Table 2 . Adverse events were observed in 68 patients $(42.5 \%)$. The more frequent adverse events were hypercholesterolemia, diarrhea, BUN/Cr elevation, hair loss, and hyperglycemia. BUN/Cr elevation was mild and resolved spontaneously or after the tacrolimus dose was adjusted in 27 of 28 patients. In one patient, BUN/Cr elevation resolved only after tacrolimus was discontinued. Most adverse events were not serious and were well-managed by adjusting tacrolimus dosage, treating the symptoms, or both. However, tacrolimus was discontinued due to adverse events in 23 (14.4\%) patients (Fig. 1). The most frequent adverse event that led to discontinuation were diarrhea $(n=13)$ and hair loss $(n=3)$. Among the 23 patients who discontinued tacrolimus due to adverse events, 17 discontinued within the first year. All adverse events that led to discontinuation resolved after the discontinuation. In addition, 13 patients stopped tacrolimus because of ineffectiveness $(n=9)$, pregnancy plan $(n=2)$, or patient refusal to continue $(n=2)$. One hundred and twenty-four people were continuously on tacrolimus, although the maintenance period differed among them.

\section{Efficacy}

Time courses of MGFA clinical classification, MGFA PIS, and
Table 2. Adverse Events of Tacrolimus

\begin{tabular}{|c|c|}
\hline Adverse event ${ }^{*}$ & $\mathrm{n}(\%)$ \\
\hline Hypercholesterolemia & $36(19.5)$ \\
\hline Diarrhea & $30(16.2)$ \\
\hline BUN/Cr elevation & $28(15.1)$ \\
\hline Hair loss & $12(6.5)$ \\
\hline Hyperglycemia & $10(5.4)$ \\
\hline Hyperphosphatemia & $8(4.3)$ \\
\hline Hypophosphatemia & $8(4.3)$ \\
\hline Iron deficiency anemia & $7(3.8)$ \\
\hline Abdominal discomfort & $7(3.8)$ \\
\hline Tremor & $5(2.7)$ \\
\hline Paresthesia & $4(2.2)$ \\
\hline Eczema & $3(1.6)$ \\
\hline Headache & $3(1.6)$ \\
\hline High blood pressure & $3(1.6)$ \\
\hline Muscle cramp & $3(1.6)$ \\
\hline Thrombocytopenia & $3(1.6)$ \\
\hline Tinea pedis, oral fungal infection & $2(1.1)$ \\
\hline Chills & $2(1.1)$ \\
\hline Hyperkalemia & $2(1.1)$ \\
\hline Hypokalemia & $2(1.1)$ \\
\hline Dizziness & $1(0.5)$ \\
\hline Myalgia & $1(0.5)$ \\
\hline Visual disturbances & $1(0.5)$ \\
\hline Dysmenorrhea & $1(0.5)$ \\
\hline Oral ulcer & $1(0.5)$ \\
\hline Nail cracks & $1(0.5)$ \\
\hline Lower extremity edema & $1(0.5)$ \\
\hline Total number of adverse events observed & $185(100.0)$ \\
\hline Number of patients with adverse events & $68 / 160(42.5)$ \\
\hline
\end{tabular}




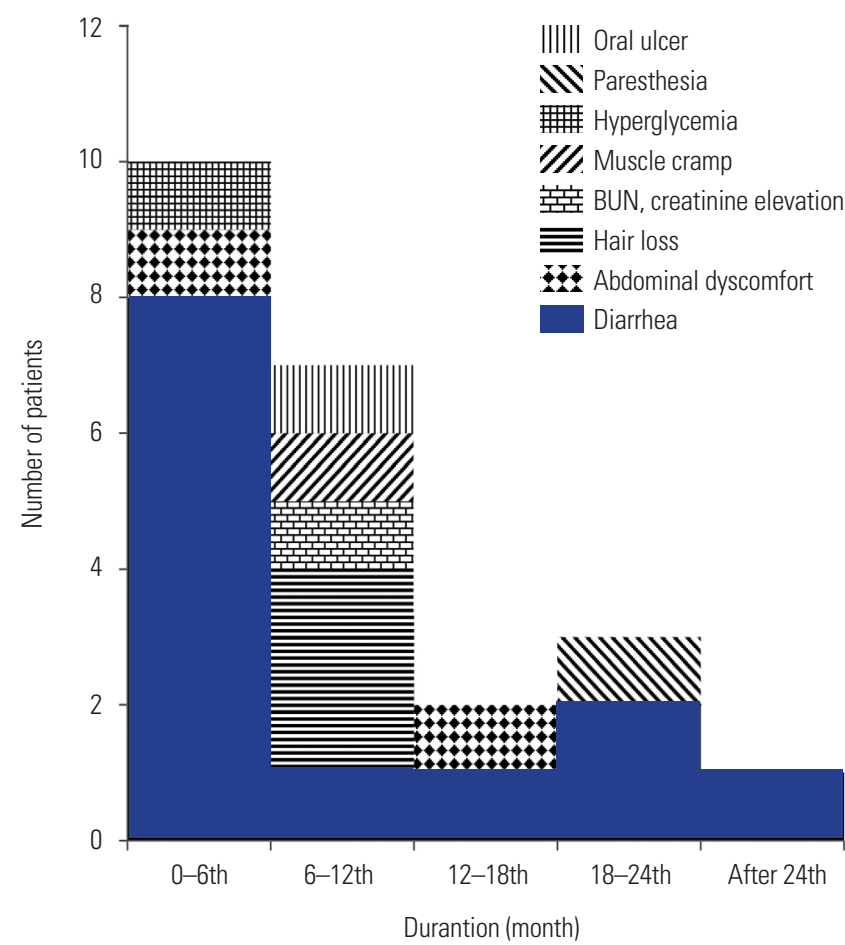

Fig. 1. Adverse effects leading to cessation of tacrolimus according to the duration of tacrolimus treatment. Twenty-three patients stopped medication due to adverse effects.

MFS are illustrated in Fig. 2. The follow-up duration of MG before initiating tacrolimus was more than 6 months in 154, 12 months in 144, 18 months in 136, and 24 months in 127 patients. The duration of tacrolimus treatment was more than 6 months in 121,12 months in 70,18 months in 53 , and 24 months in 29 patients.

The proportion of patients with MGFA clinical classification I or II was $59.4 \%$ at the initiation of tacrolimus and increased to $96.6 \%$ at the final visit. The proportion of patients with MGFA PIS complete stable remission (CSR), pharmacologic remission (PR), or minimal manifestation (MM) was $25 \%$ at the initiation of tacrolimus and increased to $96.6 \%$ at the final visit. The proportion of patients with MFS 1 or 2 was $60 \%$ at the initiation of tacrolimus and increased to $96.6 \%$ at the final visit. Mean tacrolimus maintenance period was 14.3 months at the final visit.

The mean prednisolone dose \pm standard error was $15.2 \pm 1.1$ $\mathrm{mg} /$ day at 24 months, $14.2 \pm 1.0 \mathrm{mg} /$ day at 18 months, $13.5 \pm 0.8$ $\mathrm{mg} /$ day at 12 months, and $14.6 \pm 0.9 \mathrm{mg} /$ day at 6 months before initiating tacrolimus. The mean dose of prednisolone was $21.2 \pm 1.2 \mathrm{mg} /$ day at the initiation of tacrolimus. The mean prednisolone dose was $14.2 \pm 0.7 \mathrm{mg} /$ day at 6 months, $11.8 \pm 0.6 \mathrm{mg} /$ day at 12 months, $11.3 \pm 0.8 \mathrm{mg} /$ day at 18 months, and $10.9 \pm 0.7$ $\mathrm{mg} /$ day at 24 months after initiating tacrolimus. Compared to the baseline, prednisolone dose was significantly lower at 6 , 12,18 , and 24 months of using tacrolimus $[6.95 \pm 0.89 \mathrm{mg} / \mathrm{day}$, $95 \%$ confidence interval (CI) 5.18 to $8.71 ; 9.42 \pm 0.99 \mathrm{mg} /$ day, 95\% CI 7.46 to 11.38 ; $9.93 \pm 1.18 \mathrm{mg} /$ day, $95 \%$ CI 7.59 to 12.27 ; and $10.28 \pm 1.08 \mathrm{mg} /$ day, $95 \% \mathrm{CI} 8.14$ to 12.42 ; respectively, $p<$

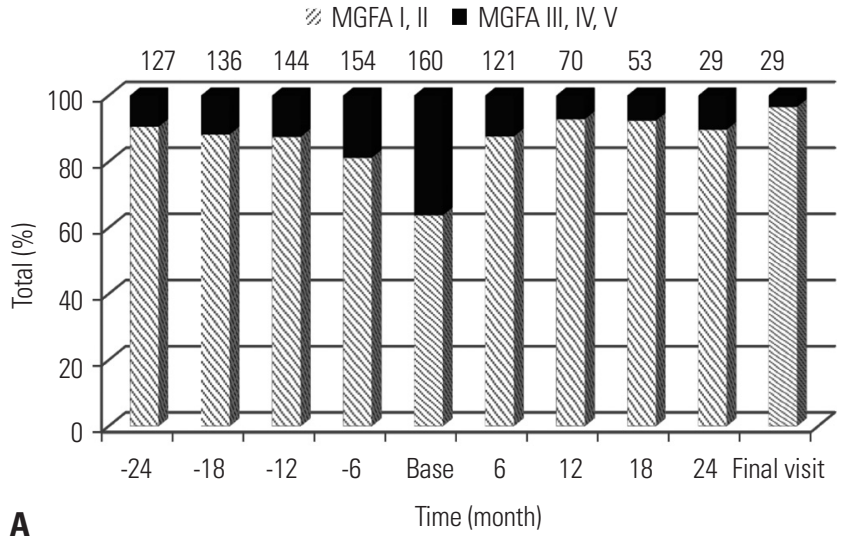

MGFA-PIS

- Improved, unchanged or worse

\# Complete stable remission, pharmachologic remission, or minimal manifestation

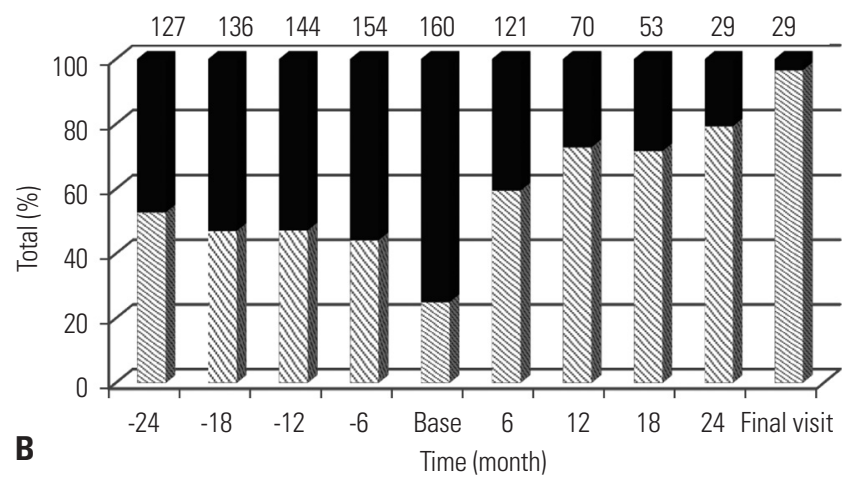

MFS $\square 1 \square 2 \square 3 \square 4 \square$

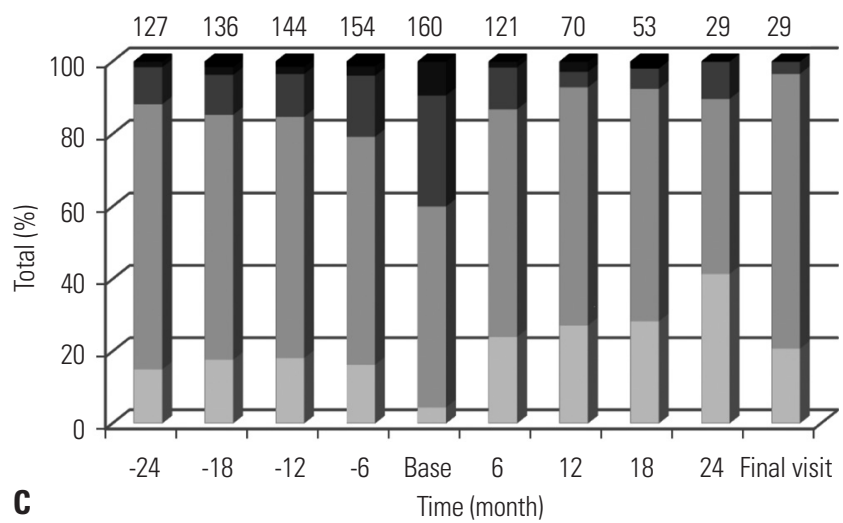

Fig. 2. MGFA clinical classification (A), MGFA-PIS (B), and MFS distribution (C) before and after tacrolimus treatment. MGFA, Myasthenia Gravis Foundation of America; MGFA-PIS, MGFA post-intervention status; MFS, Myasthenic Functional Score.

0.001]. Prednisolone dose at 6 months prior to tacrolimus and 6 months after tacrolimus did not differ significantly $(0.38 \pm$ $1.03 \mathrm{mg} /$ day, $95 \%$ CI 1.66 to 2.42; $p=0.716$ ). However, prednisolone doses decreased significantly from 6 months before initiating tacrolimus to 12 months after $(2.85 \pm 0.92 \mathrm{mg} /$ day, $95 \%$ CI 1.03 to 4.66 ; $p=0.002), 18$ months after $(3.36 \pm 0.99 \mathrm{mg} /$ day, 


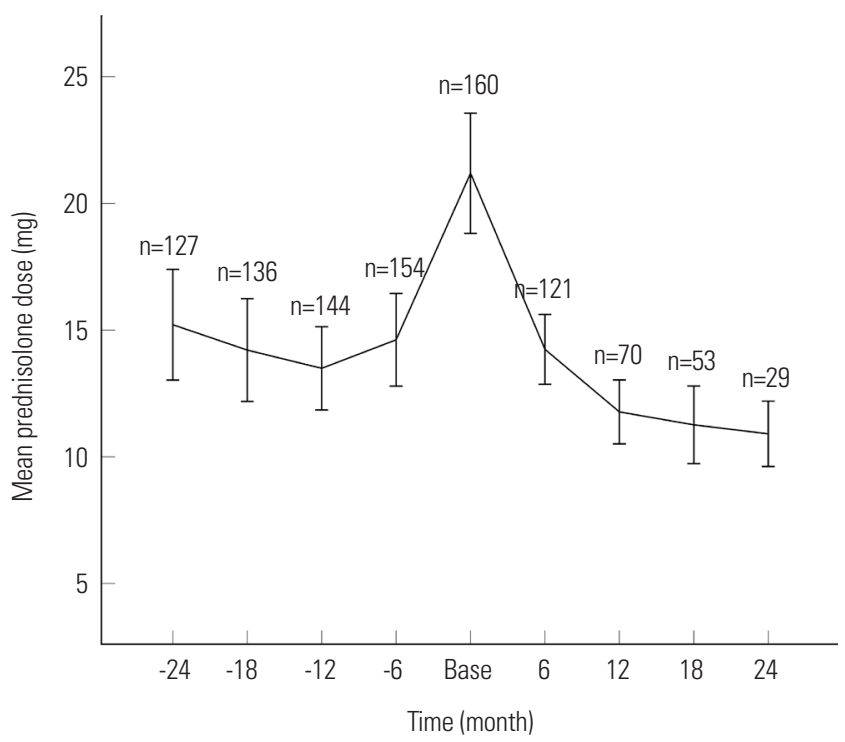

Fig. 3. Prednisolone dose before and after tacrolimus administration. Prednisolone dose while taking tacrolimus was lower than the dose before tacrolimus administration.

95\% CI 1.40 to $5.32 ; p=0.001)$, and 24 months after $(3.71 \pm 0.93$ $\mathrm{mg} /$ day, $95 \%$ CI 1.86 to 5.56; $p<0.001$ ) (Fig. 3).

\section{DISCUSSION}

In this study, we reported data from $160 \mathrm{MG}$ patients treated with tacrolimus for a mean period of 16.48 months (range 0-71 months). The mean duration from MG onset to the start of tacrolimus treatment was about 11 years. A total of 108 patients (67.5\%) had been administered other immunosuppressants, such as azathioprine, cyclosporine, and mycophenolate mofetil, which were replaced with tacrolimus. These subjects experienced repeated worsening of MG or difficulty in reducing the prednisolone dose. Therefore, most subjects in this study had a long and intractable disease course and were resistant to or dependent on prednisolone. In 105 patients (65.6\%), tacrolimus was used due to exacerbation of MG, which also required an increase in the prednisolone dose administered. Therefore, MG severity and prednisolone dose at the time of tacrolimus initiation cannot be compared with those after tacrolimus treatment, as has been done in most previous studies on tacrolimus in $\mathrm{MG}^{9,11-16,18}$ because an increase in prednisolone can influence the course of MG. In addition, the efficacy of tacrolimus can be overestimated by regression toward the mean. Therefore, we investigated MG severity and prednisolone dose for two years before and after initiating tacrolimus administration.

To evaluate the efficacy of tacrolimus, MGFA clinical classification, MGFA PIS, MFS, and oral prednisolone dose were investigated. None of the four parameters changed significantly over the two years before tacrolimus administration. At the time of tacrolimus administration, however, the proportion of patients with severe MG status and the oral prednisolone dose both increased. As the duration of tacrolimus use increased, the proportion of patients with severe MG status decreased. At two years after tacrolimus initiation, the proportions of patients with CSR, PR, or MM were significantly higher than those observed at the initiation of tacrolimus and before tacrolimus use. The oral prednisolone dose also decreased as the duration of tacrolimus use increased. The mean oral prednisolone dose was $14.6 \pm 0.9 \mathrm{mg} /$ day at 6 months before the initiation of tacrolimus, $21.2 \pm 1.2 \mathrm{mg} /$ day at the initiation of tacrolimus, $14.2 \pm 0.7$ $\mathrm{mg} /$ day at 6 months after tacrolimus treatment, and 11.8 \pm 0.6 $\mathrm{mg}$ /day at 12 months after tacrolimus treatment. At 6 months after tacrolimus treatment, the oral prednisolone dose was significantly lower than that at the initiation of tacrolimus. However, the doses between 6 months before (14.6 $\pm 0.9 \mathrm{mg} /$ day) and after $(14.2 \pm 0.7 \mathrm{mg} /$ day $)$ the initiation of tacrolimus did not differ significantly. The oral prednisolone dose at 12 months after tacrolimus treatment was significantly lower than that at 6 months before initiating tacrolimus and at the initiation of tacrolimus. These findings suggest that long-term treatment with tacrolimus may improve the clinical status and decrease the required dose of oral prednisolone in patients with MG. Considering that our MG patients had a long and intractable disease courses, the steroid-sparing effect of long-term tacrolimus therapy shown in this study is encouraging.

Most adverse events of tacrolimus in this study were not serious, similar to the results of previous studies. ${ }^{9-21}$ If necessary, tacrolimus was reduced or discontinued, and the adverse events resolved. In our study, $42.5 \%$ of patients had an adverse event (Table 2). In previous reports, the proportion of patients with adverse events varied from $0 \%$ to $87.5 \%{ }^{9-21}$ This variation in incidence of adverse events may be a result of different study designs. In a randomized, double-blind, placebo-controlled study of tacrolimus, $\mathbf{8 7 . 5 \%}$ of patients treated with tacrolimus had adverse events. ${ }^{19}$ However, the rate of adverse events was low in most retrospective studies. ${ }^{9-18}$ These results suggest that adverse events of tacrolimus rely on patient reports without systemic investigation in many retrospective studies. In this study, any adverse events that were possibly associated with tacrolimus were included, and the causal relationship between tacrolimus and adverse events was not considered. Long-term use of other medications, including prednisolone, other immunosuppressants, and pyridostigmine bromide, may have affected the rate of adverse events in this study. Although most adverse events were not serious, a substantial number of patients discontinued tacrolimus due to the adverse events, especially in the early periods of tacrolimus treatment. Therefore, physicians should pay attention to adverse events, especially in the early periods. However, the discontinuation of tacrolimus could be affected by many factors, such as the presence of alternative therapies and health insurance issues. Therefore, the rate of discontinuation may vary depending on the situation at the clinical site. 
The limitations of this study are mostly related to its retrospective study design and the nature of data collection. However, the results of this study represent one of the few published works on the use of tacrolimus for MG in a real-life setting. In this study, most patients (64\%) showed mild weakness or no symptoms at the beginning of tacrolimus administration. Additionally, information about the clinical status and oral prednisolone dose was obtained from patients who continued treatment with tacrolimus. Therefore, this study may not be free from selection bias, which would result in inclusion of data only from patients with a mild disease course and good prognosis or in whom tacrolimus was effective and led to a positive result on the steroid-sparing effect of tacrolimus. Furthermore, the patients with MG were on other immune modulating therapies and/or had thymectomy performed prior to tacrolimus. In the event of acute exacerbation, intensive treatment was applied. It should be considered that these treatments may have affected the assessment of tacrolimus efficacy in the treatment of MG, including reduction of steroid doses. Nevertheless, a substantial number of patients with MG improved after tacrolimus treatment and the oral prednisolone dose prescribed to these patients could be reduced. Considering that the subjects in this study had a long disease course and were resistant to or dependent on prednisolone over an extended period, these results suggest that tacrolimus can be an effective therapy with steroid-sparing effects for at least some patients with MG. Further studies are required to characterize patients with MG who have a favorable response to tacrolimus.

In conclusion, we deemed tacrolimus to be a safe and effective treatment in our real-world population of patients with MG. The steroid-sparing effect was demonstrated after longterm tacrolimus use.

\section{ACKNOWLEDGEMENTS}

This study was funded by Astellas Pharma Korea, Inc.

\section{AUTHOR CONTRIBUTIONS}

Yool-hee Kim, MD contributed to conceptualization, methodology, software, formal analysis, investigation, data curation, original draft preparation, review, and editing. Ha Young Shin, MD contributed to conceptualization, methodology, validation, review, and editing. Seung Min Kim, MD contributed to methodology, validation, review, and editing.

\section{ORCID iDs}

Yool-hee Kim Ha Young Shin https://orcid.org/0000-0003-2397-7845 Seung Min Kim https://orcid.org/0000-0002-4408-8265 https://orcid.org/0000-0002-4384-9640

\section{REFERENCES}

1. Lewis RA, Selwa JF, Lisak RP. Myasthenia gravis: immunological mechanisms and immunotherapy. Ann Neurol 1995;37 Suppl 1: S51-62.

2. Meriggioli MN, Sanders DB. Autoimmune myasthenia gravis: emerging clinical and biological heterogeneity. Lancet Neurol 2009;8:475-90.

3. Carr AS, Cardwell CR, McCarron PO, McConville J. A systematic review of population based epidemiological studies in Myasthenia Gravis. BMC Neurol 2010;10:46.

4. Lee HS, Lee HS, Shin HY, Choi YC, Kim SM. The epidemiology of myasthenia gravis in Korea. Yonsei Med J 2016;57:419-25.

5. Grob D, Brunner N, Namba T, Pagala M. Lifetime course of myasthenia gravis. Muscle Nerve 2008;37:141-9.

6. Schreiber SL, Crabtree GR. The mechanism of action of cyclosporin A and FK506. Immunol Today 1992;13:136-42.

7. Kino T, Hatanaka H, Hashimoto M, Nishiyama M, Goto T, Okuhara $\mathrm{M}$, et al. FK-506, a novel immunosuppressant isolated from a Streptomyces. I. Fermentation, isolation, and physico-chemical and biological characteristics. J Antibiot (Tokyo) 1987;40:1249-55.

8. Kino T, Hatanaka H, Miyata S, Inamura N, Nishiyama M, Yajima T, et al. FK-506, a novel immunosuppressant isolated from a Streptomyces. II. Immunosuppressive effect of FK-506 in vitro. J Antibiot (Tokyo) 1987;40:1256-65.

9. Evoli A, Di Schino C, Marsili F, Punzi C. Successful treatment of myasthenia gravis with tacrolimus. Muscle Nerve 2002;25:111-4.

10. Konishi T, Yoshiyama Y, Takamori M, Yagi K, Mukai E, Saida T; Japanese FK506 MG Study Group. Clinical study of FK506 in patients with myasthenia gravis. Muscle Nerve 2003;28:570-4.

11. Konishi T, Yoshiyama Y, Takamori M, Saida T. Long-term treatment of generalised myasthenia gravis with FK506 (tacrolimus). J Neurol Neurosurg Psychiatry 2005;76:448-50.

12. Nagane Y, Utsugisawa K, Obara D, Kondoh R, Terayama Y. Efficacy of low-dose FK506 in the treatment of Myasthenia gravis--a randomized pilot study. Eur Neurol 2005;53:146-50.

13. Ponseti JM, Azem J, Fort JM, Codina A, Montoro JB, Armengol M. Benefits of FK506 (tacrolimus) for residual, cyclosporin- and prednisone-resistant myasthenia gravis: one-year follow-up of an openlabel study. Clin Neurol Neurosurg 2005;107:187-90.

14. Ponseti JM, Azem J, Fort JM, López-Cano M, Vilallonga R, Buera M, et al. Long-term results of tacrolimus in cyclosporine- and prednisone-dependent myasthenia gravis. Neurology 2005;64:1641-3.

15. Ponseti JM, Azem J, Fort JM, López-Cano M, Vilallonga R, Gamez $\mathrm{J}$, et al. Experience with starting tacrolimus postoperatively after transsternal extended thymectomy in patients with myasthenia gravis. Curr Med Res Opin 2006;22:885-95.

16. Shimojima Y, Matsuda M, Gono T, Ishii W, Tokuda T, Ikeda S. Tacrolimus in refractory patients with myasthenia gravis: coadministration and tapering of oral prednisolone. J Clin Neurosci 2006;13: 39-44.

17. Tada M, Shimohata T, Tada M, Oyake M, Igarashi S, Onodera O, et al. Long-term therapeutic efficacy and safety of low-dose tacrolimus (FK506) for myasthenia gravis. J Neurol Sci 2006;247:17-20.

18. Nagaishi A, Yukitake M, Kuroda Y. Long-term treatment of steroiddependent myasthenia gravis patients with low-dose tacrolimus. Intern Med 2008;47:731-6.

19. Yoshikawa H, Kiuchi T, Saida T, Takamori M. Randomised, double-blind, placebo-controlled study of tacrolimus in myasthenia gravis. J Neurol Neurosurg Psychiatry 2011;82:970-7.

20. Zhao CB, Zhang X, Zhang H, Hu XQ, Lu JH, Lu CZ, et al. Clinical efficacy and immunological impact of tacrolimus in Chinese patients with generalized myasthenia gravis. Int Immunopharmacol 2011;11:519-24.

21. Cruz JL, Wolff ML, Vanderman AJ, Brown JN. The emerging role of tacrolimus in myasthenia gravis. Ther Adv Neurol Disord 2015;8:92- 
103.

22. Ahn SW, Joo IS, Kim BJ, Sung JJ, Kang SY, Oh J, et al. A multicenter prospective observational study on the safety and efficacy of tacrolimus in patients with myasthenia gravis. J Neurol Sci 2017;379: 271-5.

23. Jaretzki A 3rd, Barohn RJ, Ernstoff RM, Kaminski HJ, Keesey JC, Penn AS, et al. Myasthenia gravis: recommendations for clinical research standards. Task Force of the Medical Scientific Advisory
Board of the Myasthenia Gravis Foundation of America. Ann Thorac Surg 2000;70:327-34.

24. Myasthenia Gravis Clinical Study Group, Gajdos P, Elkharrat D, Chevret S, Chastang C, Raphael J, Bolgert F, et al. A randomised clinical trial comparing prednisone and azathioprine in myasthenia gravis. Results of the second interim analysis. J Neurol Neurosurg Psychiatry 1993;56:1157-63. 